\title{
A novel approach to the treatment of a calyceal diverticulum
}

\author{
Karen E. Driscoll · Christine Kim
}

Received: 8 August 2007 / Accepted: 8 August 2007/Published online: 1 September 2007

(C) Springer London 2007

\begin{abstract}
Our report details a robotically assisted laparoscopic calyceal diverticulectomy in a 14 year old female. This novel approach has not previously been described in the pediatric population. Our patient presented with hematuria and flank pain after an episode of blunt trauma. Robotically assisted laparoscopic diverticulectomy was successfully performed and the patient discharged home the following morning. Her follow-up at three months was successful, with no pain and no recurrence on ultrasound.
\end{abstract}

Keywords Calyceal diverticulum $\cdot$ Robotically assisted . Pediatric

\section{Case report}

A 15-year-old girl was evaluated for intermittent hematuria and right flank pain following a fall during a sporting activity. She had no prior history of genitourinary abnormalities. On physical examination, she exhibited some fullness in her right abdomen and flank. A computed tomography (CT) scan revealed a large cystic structure originating from the right upper and anterior portion of the kidney (see Fig. 1). A voiding cystogram revealed no vesicoureteral reflux. An intravenous pyelogram revealed a duplicated collecting system with a probable communication between the lower pole and a calyceal diverticulum (see Fig. 2). Due to its large size, the accumulation of contrast was slow and faint. Given the size of the diverticulum and its risk of rupture and infection, the family elected for surgical excision.

K. E. Driscoll $(\varangle) \cdot$ C. Kim

263 Farmington Avenue, Farmington, CT 06030-2817, USA

e-mail: kdriscoll@resident.uchc.edu
We initially performed a cystoscopy and retrograde pyelogram. This confirmed a neck of communication between the right collecting system and the large calyceal diverticulum. It was also evident that what had appeared to be a duplicated system on the IVP was in fact the calyx being displaced by the diverticulum. We placed a 4-French ureteral catheter for easier identification of the diverticular neck. The patient was then placed in a modified flank position, $30^{\circ}$ right side elevated, and secured to the bed. A transperitoneal approach was used. Using a Hassan technique, we placed a $12 \mathrm{~mm}$ port infraumbilically and two $8 \mathrm{~mm}$ robotic ports. The first robotic port was halfway between the umbilicus and the xiphoid, and the second port in the right lower quadrant along the anterior axillary line. An additional $5 \mathrm{~mm}$ port was placed in the left lower quadrant. After reflecting the colon medially, a large fluidfilled structure was seen along the lower portion of the right kidney. There was no significant parenchyma or feeding blood vessels associated with this structure. The diverticulum was opened inferiorly and the majority of the wall was excised. By injecting saline through the ureteral catheter, the diverticular neck was easily identified and oversewn with 5-0 Vicryl suture. Indigo carmine was injected and verified a watertight seal closure. The backwall of the diverticulum was left in place and was fulgurated. In addition, we secured an omental patch over the base. The excised diverticular wall was placed in an Endo-catch bag and removed through the umbilical port under direct vision. A double-J ureteral catheter and foley catheter were placed to provide maximal drainage. On postoperative day 1 , the foley catheter was removed, and the patient was discharged home.

The stent was removed one month later and a retrograde pyelogram performed. This showed a closed collecting system and no evidence of recurrence. Follow up at two 
Fig. $1 \mathrm{CT}$ of the abdomen and pelvis with IV contrast demonstrating large right calyceal diverticulum

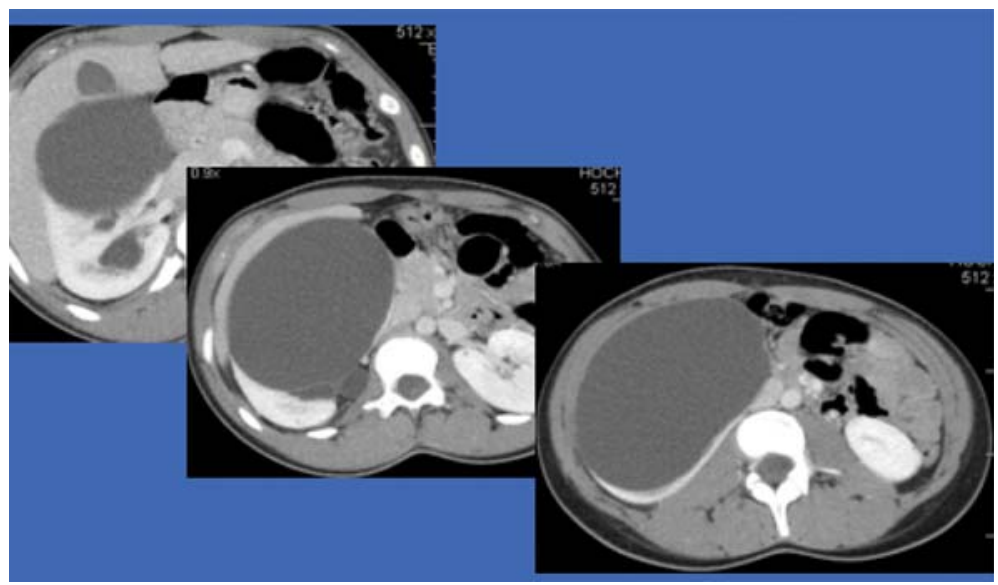

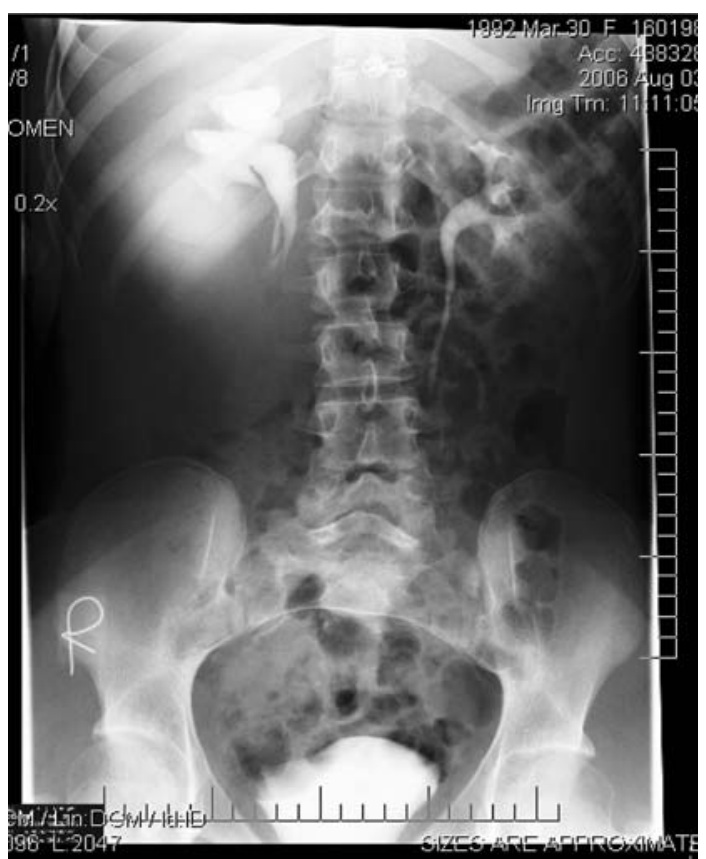

Fig. 2 IVP demonstrating blush of IVP dye into diverticulum

months shows a normal renal ultrasound with no hydronephrosis or recurrence of the diverticulum (see Fig. 3).

\section{Discussion}

Calyceal diverticula are smooth-walled, urine-filled cavities lined with transitional cell epithelium, and which communicate with the collecting system through a narrow infundibula. Although the lining is nonsecretory, the divertiulum contains urine due to passive filling from the adjacent collecting system. Clinical presentations vary and include infection, stones, and rupture. The incidence is the same in adults and pediatric patients. One study from the Mayo clinic found an incidence of 3.5 for every 1,000 IVPs

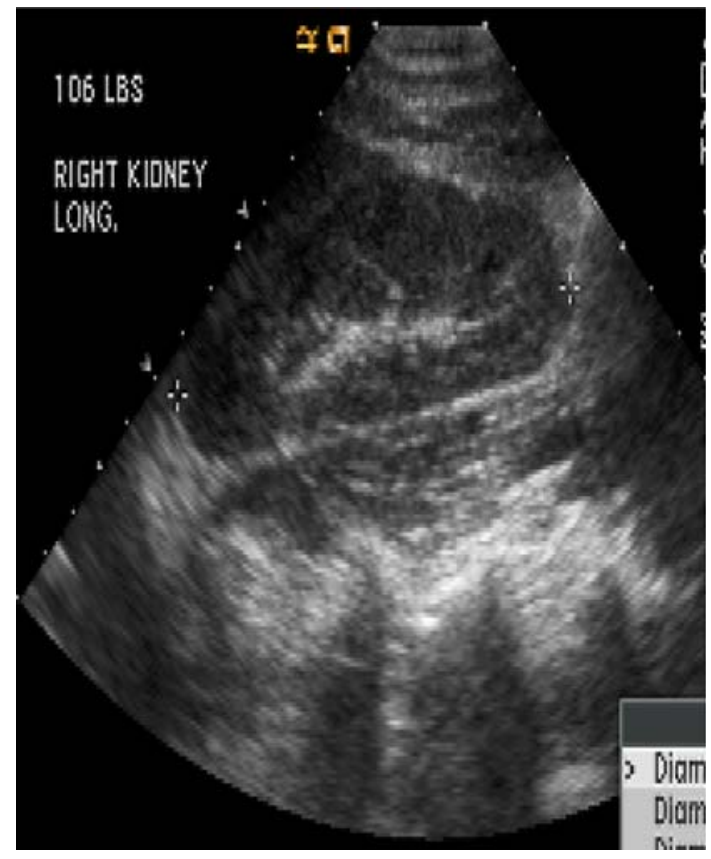

Fig. 3 Postoperative ultrasound revealing complete resolution and no hydronephrosis

performed. This study also reported vesicoureteral reflux in two-thirds of these patients with calyceal diverticulum [1].

The management of calyceal diverticula has been debated. Classically, diverticula were treated with open diverticulectomy and oversewing of the diverticular neck. Electroshock wave ithotripsy (ESWL) has a high failure rate because stones often get caught up at the neck and fail to pass. Other approaches include more aggressive use of ureteroscopy and percutaneous procedures. Difficulties encountered in the percutaneous approach include difficulty in accessing the diverticulum, bleeding, and persistent diverticula [2]. Laparoscopy is an alternative approach that has been used with favorable results. Several authors have developed algorithms in which you choose the treatment based on the location and thickness of the 


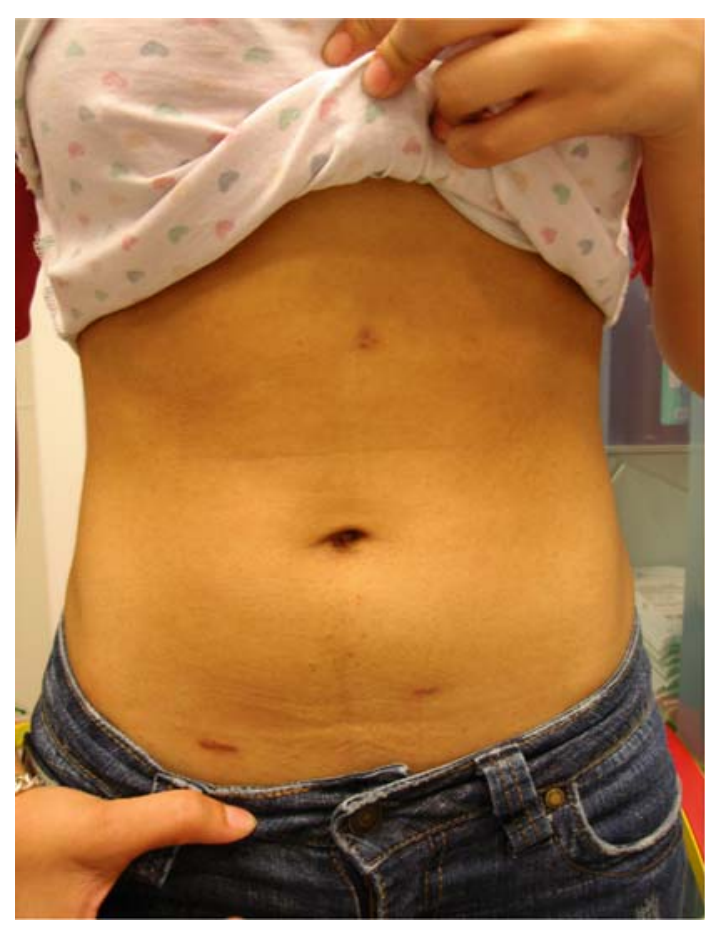

Fig. 4 Postoperative picture demonstrating the cosmesis of a robotic approach

capsule [3, 4]. Debate exists regarding which out of retroperitoneal versus intraperitoneal laparoscopic approaches are preferable [5].

The diverticulum in our patient was massive in size, and taking up a significant portion of her abdominal cavity. In addition, the patient was small and thin for her age. We felt a transperitoneal approach was most suitable in this case. It gave easy exposure to the diverticulum and adjacent structures. The combination of a watertight closure and internal drainage with a ureteral stent obviated the need for any external drains.
The majority of the literature on robotally assisted laparoscopy in pediatrics is for pyeloplasty. Benefits include improved cosmesis, decreased hospital stay, and decreased narcotic use [6]. These advantages proved evident for this patient as well. She was discharged from the hospital in less than $24 \mathrm{~h}$ and resumed normal activity in one week. Her follow-up shows resolution of the diverticulum and her cosmetic result is excellent (see Fig. 4).

\section{Conclusion}

We feel that robotically assisted laparoscopy is a useful tool in multiple pediatric surgeries. It allows excellent visualization and precise maneuvering. To our knowledge, this case reports the first pediatric robotically assisted laparoscopic calyceal diverticulectomy.

\section{References}

1. Timmons JW Jr et al. (1975) Caliceal diverticulum. J Urol 114(1):6-9

2. Schwartz BF, Stoller ML (2000) Percutaneous management of caliceal diverticula. Urol Clin North Am 27(4):635-645

3. Miller SD et al. (2002) Laparoscopic management of caliceal diverticular calculi. J Urol 167(3):1248-1252

4. Canales B, Monga M (2003) Surgical management of the calyceal diverticulum. Curr Opin Urol 13(3):255-260

5. Casale $\mathrm{P}$ et al. (2004) The pediatric caliceal diverticulum: diagnosis and laparoscopic management. J Endourol 18(7):668671

6. Lee RS et al. (2006) Pediatric robot assisted laparoscopic dismembered pyeloplasty: comparison with a cohort of open surgery. J Urol 175(2):683-687 (discussion 687) 